\title{
Von Recklinghausen neurofibromatosis and genetic linkage studies: clinical considerations
}

\author{
VINCENT M RICCARDI* AND JOHN C CAREY† \\ From * the NF Program, Baylor College of Medicine, Houston, Texas 77030; and the Genetics Division, \\ Department of Pediatrics, University of Utah Medical School, Salt Lake City, Utah, USA.
}

SUMMARY For genetic linkage purposes, a subject at risk for Von Recklinghausen neurofibromatosis (VRNF) is one who has a first degree relative with independently documented VRNF. The presence of one or more of the designated criteria establishes the phenotypic diagnosis of VRNF in a subject at risk regardless of age. The absence of all of these criteria excludes the phenotypic diagnosis for all at risk subjects over five years of age. The absence of all the criteria in subjects at risk below five years of age or the presence of equivocal or merely suggestive findings neither excludes nor substantiates the phenotypic diagnosis of VRNF; rather, that subject should be removed from the preliminary scoring and analyses of the data.

Neurofibromatosis (NF) is a fascinating disease from many vantage points. However, what makes $\mathrm{NF}$ fascinating also contributes to problems in investigating its pathogenesis and basic nature. This is especially true for genetic linkage studies. Thus, as we embark on large scale, multi-institutional NF genetic linkage projects, it is imperative to outline the relevant problems and establish standards for dealing with them.

\section{Specific issues}

\section{HETEROGENEITY}

It is now widely accepted that NF is more than one disease. From a clinical standpoint, heterogeneity has been clear for many years, ${ }^{1} 2$ but recently direct molecular genetic analyses of tumours ${ }^{3}$ and genetic linkage studies ${ }^{4}$ have confirmed this. At the least, there are three classes of NF: Von Recklinghausen neurofibromatosis (VRNF), bilateral acoustic neurofibromatosis (BANF), and other forms of neurofibromatosis that do not fit cleanly into either of the first two categories. The locus for BANF appears to be on chromosome $22 \mathrm{q},{ }^{34}$ while a locus for VRNF has been identified on $17 q{ }^{4 a}$ Thus, VRNF and BANF are mutually exclusive diagnoses. On the other hand, at least some instances of the third category of NF may eventually be seen as merely variant forms of either VRNF or BANF.

VRNF itself is clinically very variable. However, whether the clinical heterogeneity also reflects genetic heterogeneity (that is, multiple alleles or multiple loci or both) is still a matter of speculation. The high frequency of new mutations ${ }^{56}$ lends credence to the prospect of multiple alleles.

\section{EXPRESSIVITY}

Variable expressivity is a major element of the VRNF phenotype. Said another way, variable expression is a major feature of VRNF mutations. Any one subject with VRNF is quite likely to be phenotypically different from any other subject with VRNF, both from one family to another and within a given fanily. In addition, many features of NF are age related, so that itemisation of the presence or absence of VRNF features in a given person must take age into account. Further, appreciation of the full extent of the mutation's expression will depend on the diagnostic tests, including a careful physical examination by an NF expert, that are used.

Can the expression of a VRNF mutation in any one patient be so minor as to go undetected? Although this is certainly possible, as discussed in the next section, most instances of apparently 'unexpressed' VRNF mutations can be clarified by adequate physical examination, including the use of a slit lamp biomicroscope. In addition, if one distinguishes between the predecessors (parents) and descendants (offspring) of bona fide VRNF patients, minimal expressivity only very rarely confounds the diagnosis among descendants.

\section{PENETRANCE}

While non-penetrance, that is, total lack of expression, of a VRNF mutation is widely presumed to be as great as $10 \%,{ }^{5}$ well documented instances of this 
phenomenon are very rare. ${ }^{7}$ However, because of the seriously confounding influence of nonpenetrance on genetic linkage studies, every effort must be made to minimise the potential impact of this problem. At present, this can only be done by using age related diagnostic criteria, as discussed below.

\section{Von Recklinghausen NF: diagnostic criteria}

In February 1986, an NF 'task force' was assembled by the American National Institute of Neurological and Communicative Diseases and Stroke. At that meeting, diagnostic criteria for VRNF were adopted. The diagnosis of VRNF is probable, if not certain, when any two of the following are present: (1) a first degree relative with VRNF (independently documented); (2) five or more café au lait spots at least $5 \mathrm{~mm}$ in diameter for prepubertal patients, or six or more at least $15 \mathrm{~mm}$ in diameter thereafter; (3) freckling in the axillary or inguinal regions; (4) two or more neurofibromas of any type or one plexiform neurofibroma; (5) iris Lisch nodules. Additional supportive findings characteristic of VRNF include optic pathway gliomas and pseudarthrosis (or tibial/ fibular bowing). The presence of bilateral acoustic neuromas rules out the diagnosis of VRNF and favours the diagnosis of BANF.

For the purpose of providing consistent criteria for multi-institutional VRNF genetic linkage studies, the above criteria should be used for clinical determination of the presence of the VRNF mutant gene. Since genetic linkage studies inter alia use only persons with an affected first degree relative, isolated cases presenting with only one of the other criteria will not present a diagnostic problem in this particular context.

In general, there will be three types of subjects: those for whom the diagnosis of VRNF is obvious and unequivocal; those for whom there is no basis: for considering VRNF other than the fact of a $\overrightarrow{\mathrm{E}}$ affected first degree relative; and those who have suggestive, but not sufficient, findings (for example $\frac{-}{0}$ one or two café au lait spots or similar skin lesions) While the diagnosis of VRNF is ordinarily definite by one year of age, because of the need to avoid erroneous assignment of phenotype, for genetios linkage studies one should not score a subject as definitely unaffected until he or she has reached five years of age.

\section{References}

1 Riccardi VM. Neurofibromatosis: clinical heterogeneity. Curi Prob Cancer 1982;7:1-34.

${ }^{2}$ Riccardi VM, Eichner JE. Neurofibromatosis: phenotype, natu ral history and pathogenesis. Baltimore: Johns Hopkins UnN versity Press, 1986:1-305.

${ }^{3}$ Seizinger BR, Martuza RL, Gusella JF. Loss of genes o요 chromosome 22 in tumorigenesis of acoustic neuromas. Nature 1986:322:644-7.

4 Seizinger BR, Martuza RL, Gusella JF. Genetic studies i卬 bilateral acoustic neurofibromatosis. Abstracts of the Europeag Neurofibromatosis Conference, Egham, England, Februar 1987.

4a Barker D, Wright E, Nguyen K, et al. Gene for von Reckling hausen neurofibromatosis is in the pericentromeric region of chromosome 17. Science 1987;236:1100-2.

5 Crowe FW, Schull WJ, Neel JV. A clinical, pathological, genetic study of multiple neurofibromatosis. Springfield, IllinåCharles C Thomas, 1956:1-181.

${ }^{6}$ Riccardi VM, Dobson CE, Chakraborty R, Bontke C. pathophysiology of neurofibromatosis. IX. Paternal age effee on the origin of new mutations. Am J Med Genet 1984;180 $169-76$.

7 Spence MA, Bader JL, Parry DM, et al. Linkage analysis neurofibromatosis (Von Recklinghausen disease). J Med Genef. 1983;20:334-7.

Correspondence and requests for reprints to $\mathrm{Dr} \mathrm{V}$ 致 Riccardi, NF Program, Baylor College of Medicine. 1 Baylor Plaza, Houston, Texas 77030, USA.

\section{Von Recklinghausen neurofibromatosis: a linkage study of candidate and random marker genes}

ROBERT E FERRELL*, KENNETH H BUETOW†, JOHN K DARBY JUNE E EICHNER*, JEFFREY C MURRAY§, R SMITH§, MARY WAZIRI§, SUSAN HUSON\|, AND VINCENT M RICCARDII

From * the Human Genetics Program, Department of Biostatistics, University of Pittsburgh; †Fox Chase Cancer Center, Philadelphia; $¥$ Department of Genetics, Stanford University Medical School; $\$$ Department og Pediatrics, University of Iowa Hospitals and Clinics; ||Clinical Research Center, Harrow, Middlesex, UK; and Ithe NF Program, Baylor College of Medicine, Houston, Texas, USA.

SUMMARY Genotyping, using plasma proteins or DNA polymorphisms or both, was carried

Received for publication 17 March 1987 Accepted for publication 23 March 1987 out on 30 families selected through proband with Von Recklinghausen disease. The dat provide additional evidence for the exclusiono of loci on chromosomes 3 and 5, and chromo 